\title{
Start/stop Codon-like Trinucleotides (CLTs) and Extended Clusters as New Language of DNA ${ }^{\dagger}$
}

\author{
Marija Rosandić, Matko Glunčić, and Vladimir Paar* \\ Faculty of Science, University of Zagreb, Bijenička c. 32, HR-10000 Zagreb, Croatia
}

RECEIVED JULY 21, 2011; REVISED SEPTEMBER 15, 2011; ACCEPTED SEPTEMBER 16, 2011

\begin{abstract}
DNA nucleotide sequences carry genetic information of different kinds, not just coding instructions for protein synthesis. They can play a role, for example, in alternative conformations and gene regulators. The present paper introduces the extended start/stop codon-like trinucleotides (CLTs) for noncoding DNA sequences, based on trinucleotide cluster extension generated by specific single-nucleotide multiplications. Extended cluster analysis gives rise to rich information potential as a "new language" of DNA ("CLT-language"). The analysis of start/stop-CLTs extended clusters provides qualitative and quantitative differentiation and characterization of alpha satellites, as well as of other repetitive and non-repetitive noncoding sequences. As a measure of CLT extension of DNA sequences the extension factor $r$ is introduced. Start/stop CLTs enable a distinction of three segments within alpha satellite, the first and the second as wrapping sequences and the third as a linker. Within a linker there are no start/stop CLTs. On the basis of start/stop-CLTs, it is hypothesized that these noncoding sequences may be involved in the networks of gene regulators. (doi: 10.5562/cca1948)
\end{abstract}

Keywords: alpha satellites, start/stop codons, nucleosome, cluster extension, gene regulators

\section{INTRODUCTION}

The role of noncoding sequences in expressing information has received much attention, in particular as gene regulators and carriers of several messages simultaneously. ${ }^{1-8}$ On the other hand, much attention was given to alpha satellites, as intriguing noncoding arrays. ${ }^{9-14}$ Alpha satellites are approximately periodic tandems of $\sim 171$ bp monomers in centromeric/pericentromeric regions of human and other mammal chromosomes. They could be hierarchically organized into higher order periodicity patterns known as higher-order repeat (HOR) alpha satellites. The HOR copies diverge from each other by less than $5 \%$, while alpha satellite copies within any HOR copy diverge from each other by $\sim 20$ $35 \%$ and divergence between alpha satellites outside of HORs is $20-40 \%{ }^{13}$ Unequal crossing over, restricted to tandem sequences, explains the generation and local homogenization of HOR units and accounts for large size variation among HORs on homologous chromosomes. HORs are particularly interesting since they, due to more recent evolution and by the process of unequal crossing over, enabled a rapid evolutionary progress. The available sequencing of centromeric region is still incomplete and HORs of human chromosoms are not completely sequenced.

\section{METHODS}

Identification and analysis of repeats and HORs and their consensus sequences is performed using the GRM (Global Repeat Map) algorithm ${ }^{31-33}$ applied to the Build 37.2 and Build 2.2 assemblies of human and chimpanzee genomic sequences, respectively. GRM gives a direct mapping of symbolic DNA sequences into frequency domain providing a global map of repeats. An extended codon-like trinucleotide (CLT) analysis of genomic sequences is performed using the ECLT algorithm developed in this work. Full Methods and GRM and ECLT algorithms are freely available at request.

\section{RESULTS AND DISCUSSION}

In this paper a new approach of codon-like trinucleotides (CLTs) extensions, in particular for noncoding analogs of start/stop codons, is introduced to analyze the structure of noncoding sequences. This unusual viewpoint of terminators in noncoding sequences, in terms of CLTs, is resulting from detailed analysis of dinucleotides and trinucleotides in alpha satellites, presented in this paper. As a case study, this new method is applied to the following DNA sequences: to human and chim-

\footnotetext{
$\dagger$ This article belongs to the Special Issue Chemistry of Living Systems devoted to the intersection of chemistry with life.

* Author to whom correspondence should be addressed. (E-mail: paar@hazu.hr)
} 
panzee alpha satellite consensus HORs, to monomeric alpha satellite regions and to no-repeat DNA regions (Table 1 and Supplementary table S1). In these studies the consensus sequences are determined by GRM analy$\operatorname{sis}^{25-33}$ of the recent Build 37.2 assembly (Figure 1).

First the dinucleotide frequencies in consensus alpha satellites are analyzed (Supplementary table S2). Previously, the pattern of dinucleotides and trinucleotides in noncoding sequences was extensively studied. ${ }^{15-24}$ Two classes of alpha satellites are shown here: the poly-T class (with dominant TT dinucleotides), and the poly-A class (with dominant AA). It is seen here that all consensus HOR alpha satellites are of poly-T class, while the monomeric alpha satellites are of poly-A or poly-T class. Dinucleotide frequencies in human alpha satellite consensus HORs are shown in Figure 2. Earlier investigations have found a low frequency of TA dinucleotides in large segments of noncoding DNA. Here it is found that the TA-low pattern is most pronounced in HOR alpha satellites (Figure 2), which is not expected in light of A,T-rich character of alpha satellites. On the other hand, in no-repeat noncoding sequences the TA-low effect is much less pronounced.

Analysis of frequencies of trinucleotides in alpha satellite HORs is performed for consensus HORs in human chromosomes 1, 4, 5, 7, 8, 9, 10, 11, 17, 19, X, and Y (Table 1 and Supplementary tables S1 and S3). As an illustration, ten most frequent trinucleotides in 16mer alpha satellite HORs in human chromosome 7 are shown in Table 2, in comparison to previously computed ten most frequent trinucleotides in the genome of C. elegans ${ }^{34}$ as comparison between evolutionary distant genomes. Out of ten most frequent trinucleotides in 16 mer HOR in human chromosome 7, six are present in the genome of $C$. elegans too. The remainnig four trinucleotides in these sequences differ.

Different human and chimpanzee sequences from Table 1 are characterized by domination of TTT (poly-T class) or of AAA (poly-A class).

Inspired by much interest in gene regulators, ${ }^{4-7}$ here the trinucleotides corresponding to the start (ATG) and stop (TGA, TAG, TAA) codons (CLTs) are analyzed in noncoding sequences. In each alpha satellite the two to four start- and ten to fourteen stop-CLTs are found (Figure 3 and Supplementary figure 1). The largest frequency is associated with the stop-TGA CLT (seven to ten). The main result of this study is the discovery that the CLTs are extended to cluster organization. This is illustrated in Table $3 \mathrm{a}$ for human consensus HORs.

In all alpha satellite monomers, the start-ATG CLT appears at two positions, separating monomers into two nearly equal parts. The first start-ATG CLT has always one A nucleotide overlap with the following stop-TGA CLT, thus forming the segment TGATG
(Figure 3). It could be related to fusion of the last nucleotide of one alpha satellite monomer with the starting nucleotide of the next. The second start-ATG CLT is extended and overlapped to form ATTTGGA, representing a fusion of ATTTG and TTTGGA originating from extended start-ATG and stop-TGA CLTs. The extended stop-TAA CLT appears in all HOR alpha satellites only once, at the same position, as extended TAAAAA. The only stop-CLT which appears at three positions in alpha satellites in non-extended form is TAG.

It is found here that the stop-TGA CLT extension in sex chromosomes, human $\mathrm{X}$ and $\mathrm{Y}$ and chimpanzee $\mathrm{Y}$, is twice smaller than in most of somatic chromosomes. Similarly, the investigated somatic chromosome 7 has by a factor of two reduced extension with respect to most of other human somatic chromosomes. Additionally, in alpha satellite HOR from human Y chromosome and in non-HOR poly-T alpha satellites in pericentromeric building blocks from chromosome 7 there appears the third start-ATG CLT between the two previous stop-CLTs (Figure 3). This contributes to a difference between human and chimpanzee $\mathrm{Y}$ chromosomes (Table 1).

It should be noted that small and seemingly insignificant differences in a nonlinear system of genes and regulators, as for example one additional start-ATG CLT in human Y chromosome with respect to chimpanzee $\mathrm{Y}$ chromosome, could produce significant functional differences.

Among the other CLTs, the largest frequencies and extensions are associated with CTT and TCC, but still by a factor of two smaller than the dominant extension of stop-TGA CLT. Extended cluster analysis shows a high degree of specificity for each alpha satellite monomer. Such a rich potential of specification enabled by rather high divergence between alpha satellite monomers of $\sim 20-40 \%$, generated by the mechanism of extended CLTs, reveals a deeper structural organization.

All analyzed sequences have similar summary percentages of nucleotides forming start/stop-CLTs and percentages of extended start/stop-CLTs, embedded into $\sim 60$ bp C-free subsequence of alpha satellites (Table 1). This reveals a dominant influence of A,T-rich pattern. The TA dinucleotide, due to small frequency, has no significant impact on the results. Accordingly, the A,T-rich and TA-poor patterns do not show any differentiation between sequences, including both HOR and monomeric alpha satellites.

What significantly distinguishes these alpha satellite sequences from non-alpha satellite HORs and norepeat sequences are the specificity and the level of extension of start/stop-CLTs, with dominant contribution from stop-TGA CLT (Table 1 and 3a). The largest extension of stop-TGA CLT in alpha satellites within 
Table 1. Extended and non-extended stop-TGA and summary start/stop-CLTs in selected repeat and no-repeat sequences in human and chimpanzee chromosomes, expressed in percentages rounded off at the closest integer. Chromosomes without specification of species are human, while chimpanzee chromosomes are denoted by the notation chimp. The corresponding results for start-ATG, stop-TAA, and stop-TAG CLTs are given in Table S1

\begin{tabular}{|c|c|c|c|c|c|c|c|c|c|c|}
\hline \multicolumn{4}{|c|}{ Sequence } & \multicolumn{3}{|c|}{ Start/Stop CLTs } & \multicolumn{3}{|c|}{ TGA } & \multirow[b]{2}{*}{$r$-class ${ }^{(\mathrm{i})}$} \\
\hline Chr. & Structure & P.R.U. (a) & $\begin{array}{l}\text { Poly-A/ } \\
\text { Poly-T }{ }^{(b)}\end{array}$ & $\begin{array}{c}\mathrm{nt} \\
\text { all } \mathrm{E} \& \mathrm{NE}^{(\mathrm{c})}\end{array}$ & all $\mathrm{E}^{(\mathrm{d})}$ & $\begin{array}{c}\mathrm{nt} \\
\mathrm{E} \& \mathrm{NE}^{(\mathrm{e})}\end{array}$ & $\begin{array}{l}\mathrm{nt} \\
\mathrm{E}^{(\mathrm{f})}\end{array}$ & $\begin{array}{c}\mathrm{nt} \\
\mathrm{NE}^{(\mathrm{g})}\end{array}$ & $r^{(\mathrm{h})}$ & \\
\hline 1 & $11 \mathrm{mer}$ HOR & alpha $^{(j)}$ & $\mathrm{T}$ & 41 & 66 & 23 & 19 & 4 & 5 & II \\
\hline 4 & 13mer HOR & alpha & $\mathrm{T}$ & 37 & 71 & 22 & 20 & 1 & 17 & I \\
\hline 5 & 13mer HOR & alpha & $\mathrm{T}$ & 42 & 66 & 24 & 22 & 2 & 12 & I \\
\hline 7 & 16mer HOR & alpha & $\mathrm{T}$ & 43 & 69 & 23 & 22 & 2 & 14 & I \\
\hline 8 & $11 \mathrm{mer}$ HOR & alpha & $\mathrm{T}$ & 41 & 63 & 24 & 22 & 2 & 11 & I \\
\hline 9 & 7mer HOR & alpha & $\mathrm{T}$ & 40 & 62 & 22 & 21 & 2 & 14 & I \\
\hline 10 & 18mer HOR & alpha & $\mathrm{T}$ & 37 & 71 & 15 & 13 & 1 & 11 & I \\
\hline 11 & 12mer HOR & alpha & $\mathrm{T}$ & 40 & 68 & 21 & 18 & 3 & 7 & II \\
\hline 17 & 14mer HOR & alpha & $\mathrm{T}$ & 37 & 61 & 21 & 18 & 3 & 6 & II \\
\hline 19 & 13mer HOR & alpha & $\mathrm{T}$ & 41 & 65 & 24 & 22 & 2 & 13 & I \\
\hline 19 & 17mer HOR & alpha & $\mathrm{T}$ & 43 & 68 & 23 & 22 & 2 & 13 & I \\
\hline$X$ & 12mer HOR & alpha & $\mathrm{T}$ & 41 & 66 & 23 & 20 & 3 & 7 & II \\
\hline $\mathrm{Y}$ & 45mer HOR & alpha & $\mathrm{T}$ & 44 & 71 & 25 & 22 & 3 & 8 & II \\
\hline chimp Y & 30mer HOR & alpha & $\mathrm{T}$ & 46 & 69 & 26 & 23 & 3 & 8 & II \\
\hline chimp 7 & mon. ${ }^{(\mathrm{k})}$ & alpha & $\mathrm{T}$ & 48 & 68 & 25 & 21 & 4 & 5 & II \\
\hline 7 & mon. & alpha & $\mathrm{T}$ & 40 & 71 & 21 & 20 & 1 & 17 & I \\
\hline 7 & mon. & alpha & $\mathrm{T}$ & 47 & 70 & 24 & 23 & 2 & 15 & I \\
\hline 7 & mon. & alpha & A & 44 & 65 & 20 & 16 & 4 & 5 & II \\
\hline 7 & mon. & alpha & A & 44 & 65 & 20 & 16 & 4 & 5 & II \\
\hline 1 & mon. & alpha & A & 51 & 62 & 23 & 19 & 4 & 5 & II \\
\hline 11 & mon. & alpha & $\mathrm{T}$ & 46 & 69 & 24 & 20 & 4 & 5 & II \\
\hline Y & 3mer HOR & $1.6 \mathrm{~kb}$ & A & 36 & 59 & 12 & 8 & 4 & 2 & III \\
\hline $\mathrm{Y}$ & 5mer HOR & $2.4 \mathrm{~kb}$ & $\mathrm{~T}$ & 48 & 63 & 12 & 10 & 2 & 5 & II \\
\hline $\mathrm{Y}$ & 3mer HOR & $0.55 \mathrm{~kb}$ & $\mathrm{~T}$ & 52 & 64 & 13 & 11 & 2 & 7 & II \\
\hline 5 & no-repeat ${ }^{(\mathrm{l})}$ & - & $\mathrm{T}$ & 45 & 64 & 11 & 9 & 3 & 3 & III \\
\hline 21 & no-repeat & - & A & 47 & 63 & 12 & 9 & 3 & 3 & III \\
\hline 7 & no-repeat & - & $\mathrm{T}$ & 44 & 63 & 12 & 9 & 3 & 3 & III \\
\hline 1 & $\operatorname{random}^{(\mathrm{m})}$ & - & $\mathrm{T}$ & 41 & 60 & 10 & 7 & 3 & 2 & III \\
\hline 7 & random & - & $\mathrm{T}$ & 44 & 58 & 10 & 7 & 3 & 3 & III \\
\hline
\end{tabular}

\footnotetext{
(a) Primary repeat unit.

(b) Type of sequence: A (Poly-A type), T (Poly-T type).

(c) Percentage of nucleotides in all extended and non-extended start/stop-CLTs in genomic sequence.

(d) Percentage of extended start/stop-CLTs with respect to all extended and non-extended start/stop-CLTs in genomic sequence.

(e) Percentage of nucleotides in extended and non-extended stop-TGA CLTs in genomic sequence.

(f) Percentage of nucleotides in extended stop-TGA CLTs in genomic sequence.

(g) Percentage of nucleotides in non-extended stop-TGA CLTs in genomic sequence.

(h) Quotient of nt E and nt NE.

(i) Classification of genomic sequence according to the $r$-value into three classes (see the text).

(j) Alpha satellite monomer.

(k) Monomeric alpha satellites.

(l) Illustrative $21 \mathrm{~kb}$ genomic sequences without repeats from human chromosomes 5 (contig NW_003315920.1, 23100-44100 bp), chromosome 7 (contig NW_003315922.1, 1-21000 bp), and chromosome 21 (contig NT_113952.1, 89250-110250 bp).

(m) Illustrative random sequences with nucleotide abundances the same as in alpha satellite HORs in chromosomes 1 and 7.
} 
Table 2. Ten most frequent trinucleotides in consensus $16 \mathrm{mer}$ HOR in human chromosome 7 and comparison to the computation $^{34}$ of ten most frequent nucleotides in the genome of C. elegans

\begin{tabular}{cccc}
\hline \multicolumn{2}{c}{ Human HOR in chr. 7} & \multicolumn{2}{c}{ C. elegans genome $^{34}$} \\
Trinucleotide & Frequency $^{\text {(a) }}$ & Trinucleotide & Frequency $^{\text {(a) }}$ \\
\hline TTT & 100 & AAA & 100 \\
AAA & 74 & TTT & 100 \\
GAA & 71 & ATT & 60 \\
CTT & 69 & AAT & 60 \\
TTG & 61 & GAA & 45 \\
TCT & 58 & TTC & 45 \\
TTC & 54 & CAA & 40 \\
TGA & 50 & TTG & 40 \\
AGA & 48 & TCA & 36 \\
AAC & 46 & TGA & 36 \\
\hline
\end{tabular}

${ }^{(a)}$ Expressed relatively to the most frequent trinucleotide (normalized to 100).

HORs and in monomeric alpha satellites is mostly due to selective successive multiplications of $\mathrm{T}$ nucleotides (Table 3b), and less due to the multiplications of $\mathrm{A}$ nucleotides, resulting in poly- $\mathrm{T}$ or less frequently in poly-A alpha satellites. In HORs with poly-T consensus there are also some rare cases of individual poly-A alpha satellites.

The extensions of start-ATG and stop-TAA CLTs are significantly smaller than extensions of stop-TGA CLT, and the extension of stop-TAG CLT is absent or very small in all monomeric and HOR alpha satellites (Supplementary table S1). The stop-TAA CLT has a significant extension, but is of very low frequency. On the other hand, the extension of stop-TAG CLT is sizably larger in some non-alpha satellite HORs, which have primary repeat units of $1.6,2.4$, and $0.55 \mathrm{~kb}^{32,33}$ sizably larger than alpha satellites $(\sim 0.171 \mathrm{~kb})$.

As a measure of the CLT-extension of genomic sequences the corresponding extension factor $r$ is introduced here (for definition of the factor $r$ see caption to Table 1). Accordingly, the $r$-classification of genomic sequences is defined as follows:

$\begin{array}{ll}\text { class I } & \text { for } r>8, \\ \text { class II } & \text { for } 4 \leq r \leq 8, \\ \text { class III } & \text { for } r<4 .\end{array}$

Examples of these classes are (Table 1):

TGA class I: alpha satellite HOR in human chromosome 7 ,

TGA class II: alpha satellite HOR in human chromosome X,

TGA class III: no-repeat segment in human chromosome 21.

From these analyses the basic role of stop-TGA CLT is seen: the poly-T stretch preceding $\mathrm{G}$ nucleotide and the poly-A stretch following it (poly-T $-\mathrm{G}-$ poly-A) exhaust about $30 \%$ of all $\mathrm{T}$ and $\mathrm{A}$ nucleotides in alpha satellites. The TA is bound to stop-TAA and stop-TAG (non-extended and extended) CLTs having small frequency and extension with respect to stop-TGA CLT. Only one TA is present in the $110 \mathrm{bp}$ segments of alpha satellites outside of the start/stop-CLTs and their extensions. This results in TA-low pattern of alpha satellites (Figure 2).

In alpha satellite HORs from human chromosomes 1,11 and 17 the extension of start/stop- CLTs is by a factor of two smaller than in other somatic human chromosomes, i.e., similar as in human and chimpanzee sex chromosomes (Table 1). It could be hypothesized that in these segments of chromosomes the evolutionary changes were slower than in other somatic human chromosomes.

Chromosome 10 has a smaller number of stopTGA CLTs, but their extension is similar as in other human somatic chromosomes.

Different multiplication patterns of individual A, T, G nucleotides in extension of start/stop-CLTs in alpha satellites might influence expression of codons in coding sequences. For example, stop-TGA CLT, which is most strongly extended within the alpha satellites, takes the form TTTGGAAA. We hypothesize that in this way it might exert the stop influence on some segments of the genome, possibly on codons underlying the extended stop-TGA CLT (Table 3c).

It is interesting to compare the concept of codonlike trinucleotides extension, introduced here, to the concept of Shannon N-gram extension used for reconstruction of the most likely sequence pattern by fusing overlapping triplets that was used for no-repeat sequences. ${ }^{34,35}$ It was shown that Shannon's fusing can generate motifs like TTTTCGAAAA and TTTTTGAAAA ${ }^{34,35}$ with a periodic structure on both sides of central motif, representing a nucleosome positioning patterns. Although a string similar to central motif starting with

Table 3. Illustrations of CLT extensions in alpha satellites. For description see the text.

$$
\begin{array}{ll}
\text { a } & \text { TGA } \rightarrow \text { TTGAA } \rightarrow \text { TTTTGA } \rightarrow \text { TTTTGAAA } \rightarrow \text { TGGA } \rightarrow \text { TTTGGA } \rightarrow \text { TTTGA } \rightarrow \text { TGGAAAA } \\
\text { b } & \text { T } \rightarrow \text { TT } \rightarrow \text { TTT } \rightarrow \text { TTTT } \ldots \\
\text { c } & \text { TTTGGAAA } \rightarrow \text { TTT, TTG, TGG, GGA, GAA, AAA }
\end{array}
$$



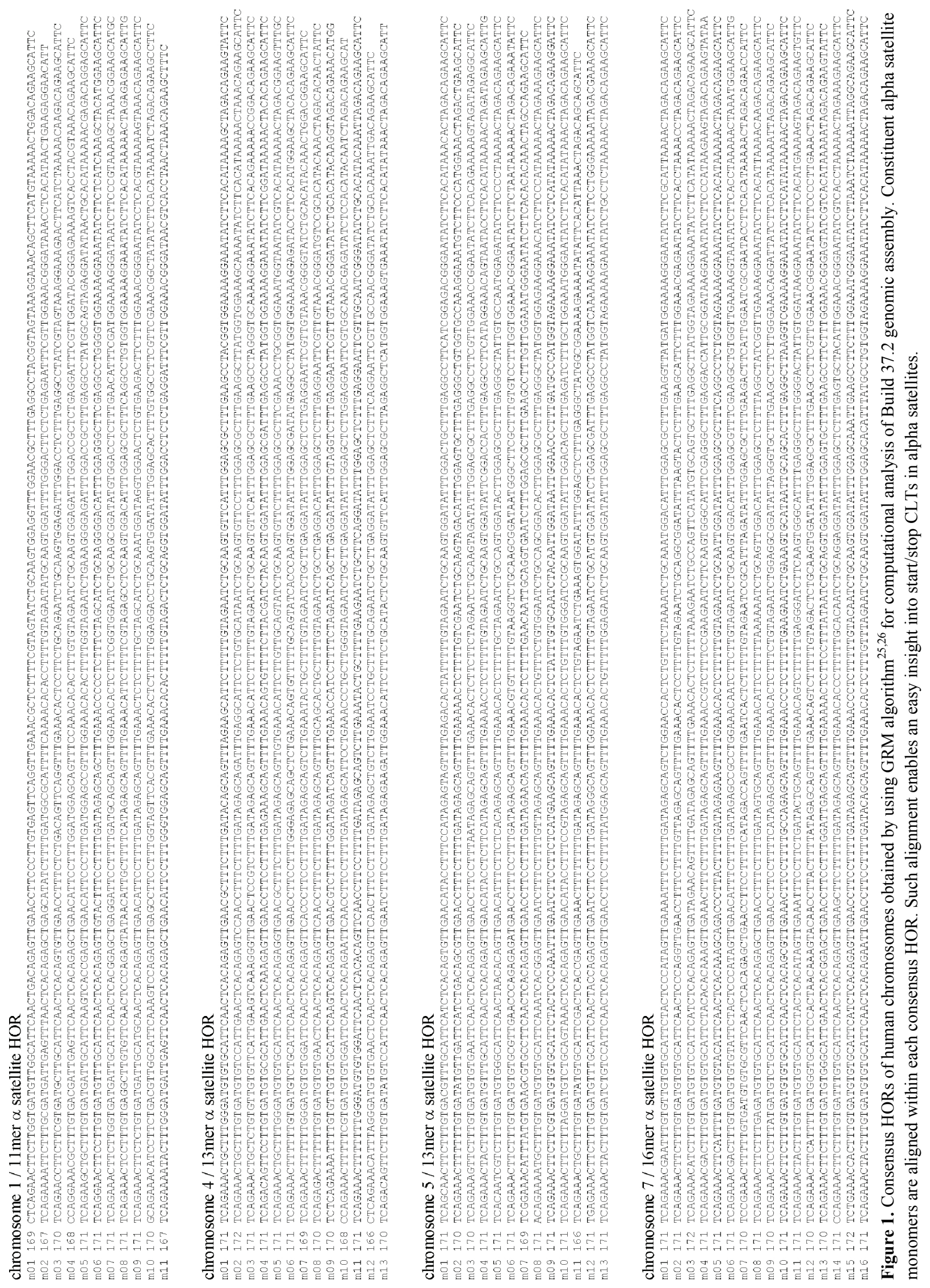

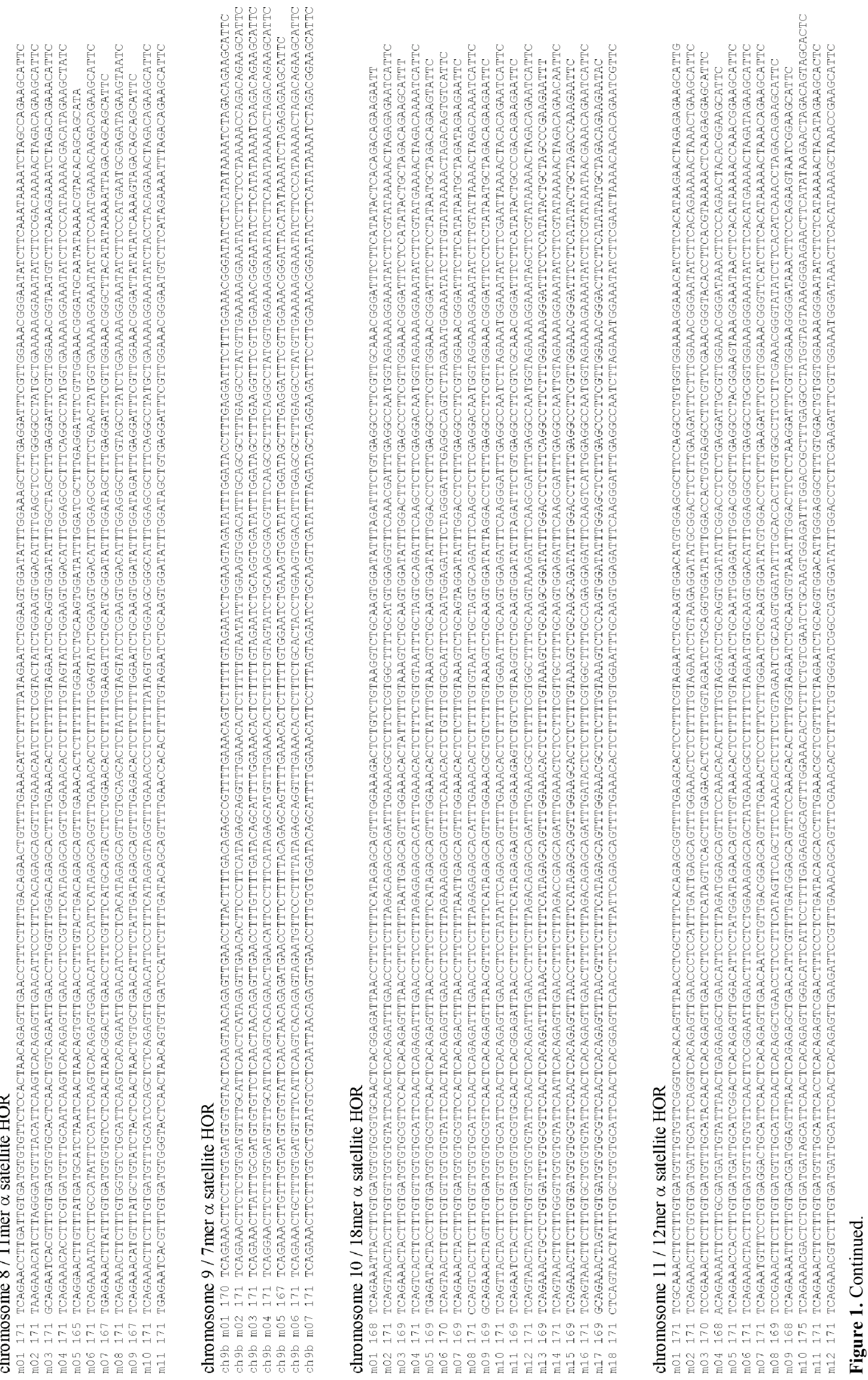

Croat. Chem. Acta 84 (2011) 331. 


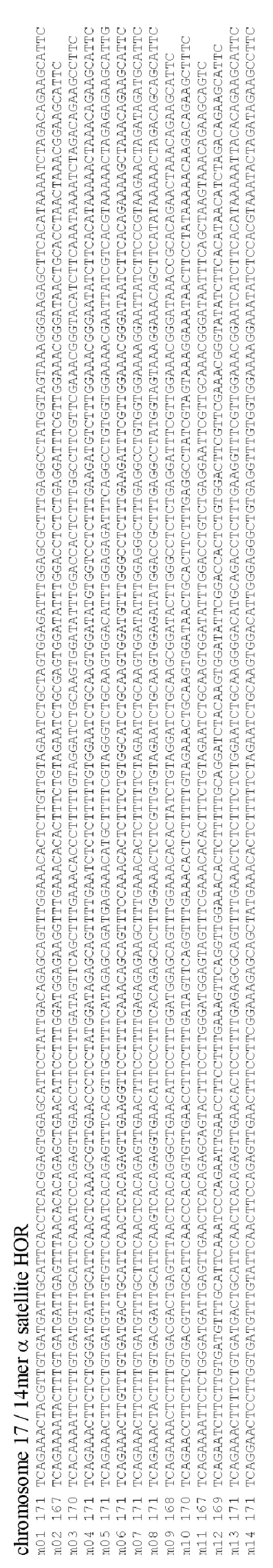

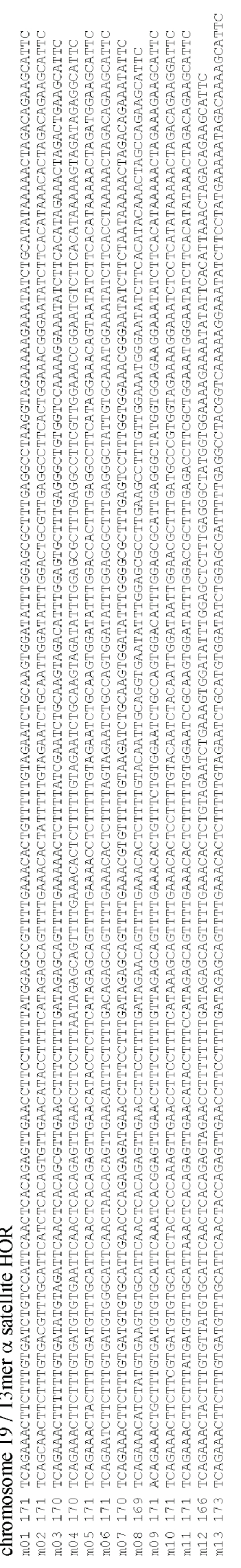

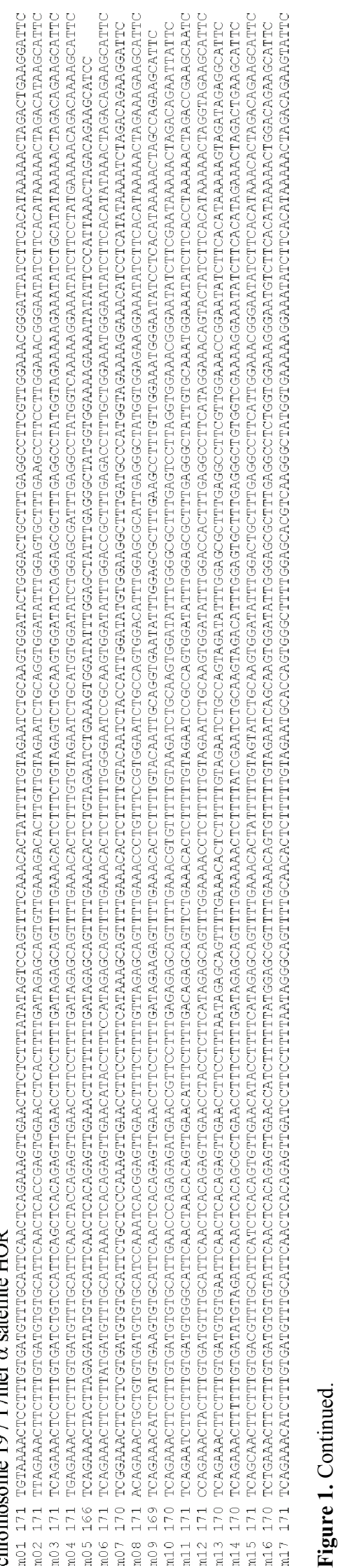



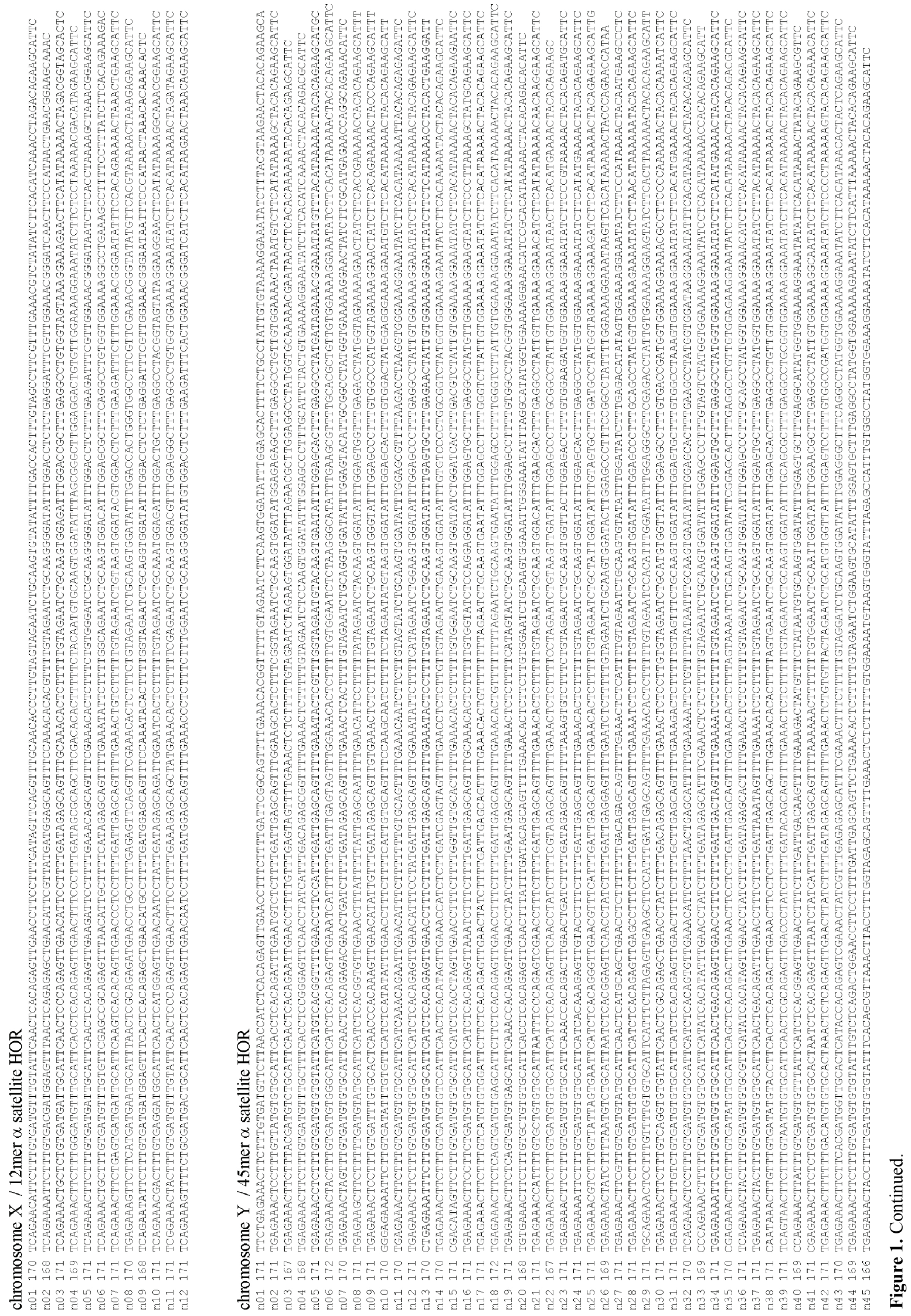

Croat. Chem. Acta 84 (2011) 331. 


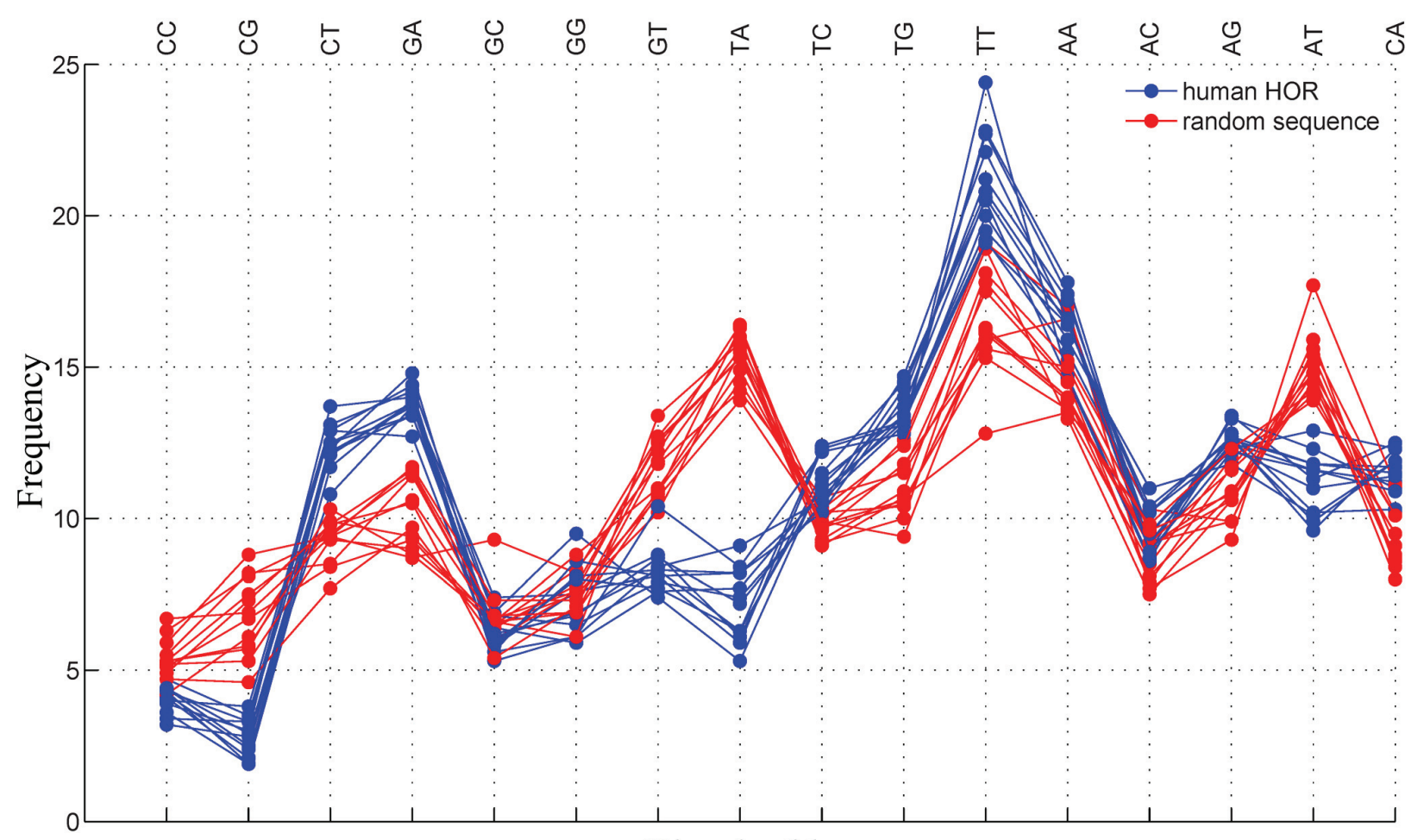

Dinucleotide

Figure 2. Dinucleotide frequencies in human consensus HOR alpha satellites and comparison to random sequences. Frequency of dinucleotides are shown for HOR alpha satellites (red) and for the corresponding random sequences (blue). It is seen that the TAlow effect is strongly pronounced in alpha satellite HORs.

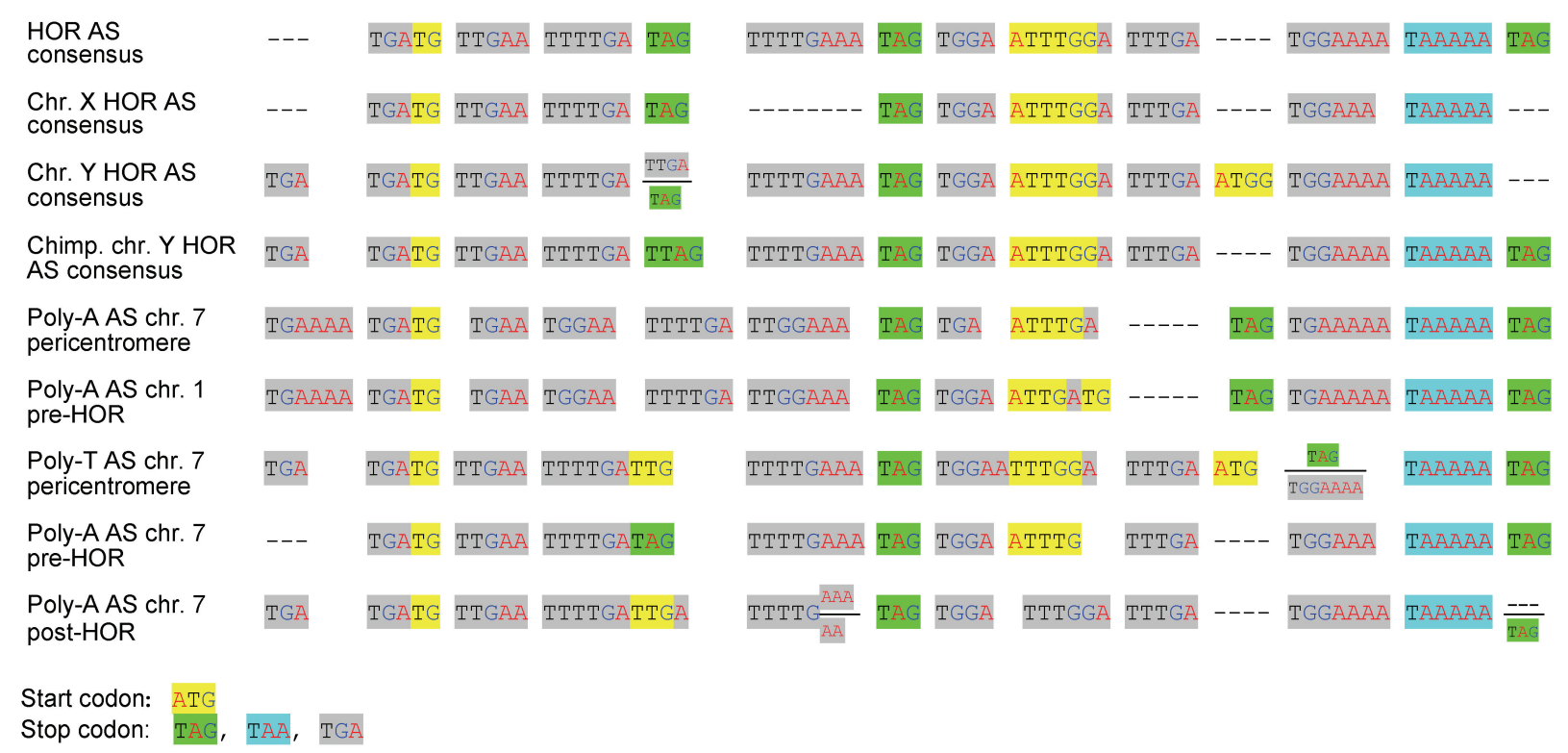

Figure 3. Specific composition of start/stop CLTs in alpha satellite monomers from HOR and monomeric sequences in human chromosomes and in chimpanzee Y chromosome. Chromosomes without specifications of species are human, while chimpanzee chromosome is denoted as chimp. AS denotes alpha satellite.

Shannon construction of trinucleotide frequencies alpha satellite HORs is found here, they do not exhibit any periodic structure to sides of the central motif (Supplementary figure 2). Here an alternative model of wrap- ping around nucleosome is proposed, based on a twofold role of start/stop CLTs, both as a component of regulatory element and as construction element of nucleosome structure. 
In the framework of this approach the structure of alpha satellite monomer is organized in three segments (Figure 4): the first ( $\sim 84 \mathrm{bp})$ and the second ( $\sim 58 \mathrm{bp})$ wrapping around the nucleosome and a linker $(\sim 29 \mathrm{bp})$. On the basis of start/stop CLTs it is recognized that the start of the first wrapping is the first ATG start-CLT in the sequence of 171-bp alpha satellite monomer, and the start of the second wrapping is the second ATG CLT (in extended form ATTTGG) positioned precisely at the half of alpha satellite monomer. The first and the second wrapping sequence end with the stop-TAG CLT. After the second wrapping sequence follows the linker of $\sim 29$ $\mathrm{bp}$, which ends with TG nucleotide. This dinucleotide is combined with ATG as the start of the next alpha satellite monomer. In this way the string TGATG is formed. As already mentioned, the TGATG represents stopTGA and start ATG CLT with A-nucleotide overlap. It is demonstrated that the first 27 bases in the linker do not contain any start/stop CLT, what significantly distinguishes linker from the two wrapping sequences in alpha satellite monomers.

There is a question why so much effort should have been spent during evolution exclusively for building elements such as sophisticated chromosome specific HORs with highly specific constituent alpha satellites. Previously we suggested that this HOR specificity and form of DNA folding is necessary that particular microtubules be bound to centromere in the corresponding chromosomes. ${ }^{31}$ Of course, the centromere should be substantially better organized and stronger so that it does not break to endanger very complex organisms such as primates and in particular Homo sapiens as the last and most sophisticated in the evolutionary chain. One might ask whether it is needed to use such a complex structure as HORs, instead of having simple microsatellite tandems without any chromosome specificity. Therefore, it could be argued that HORs may also play some regulatory roles in the network of genes and gene regulators.

Furthermore, the central position of alpha satellite HORs in chromosomes, deeply protected within specific structure of centromeres, points to their possible importance and potential influence on the whole DNA. This might be compared, in principle, with a biological organization present on macro-plan, for example, by pituitary gland as master gland, which is strongly protected in the "armour" of sella turcica in sphenoid bone of scull and has a dominant influence on all endocrine glands in the body at significant distances. CLTs having different lengths of extension give, among others, possibilities of significantly higher number of combinations and have sizably higher information potential, contributing to higher interspecies variability. In general one might argue that the presence of internal extended cluster organization of alpha satellites could enable detailed analysis and holistic approach for the whole genome.

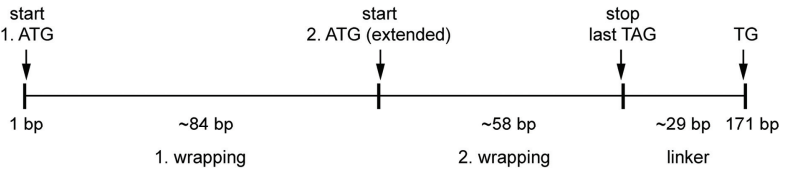

Figure 4. Start/stop CLTs scheme of wrapping alpha satellite around nucleosome and linker.

\section{CONCLUSION}

Extended start/stop codon-like clusters for noncoding sequences are introduced as a "new language" of DNA with rich information potential and shown to be most pronounced in alpha satellite higher order repeats, with a possible role in gene regulators.

This work shows in alpha satellite HORs an extended cluster organization based on $\mathrm{T}$ and/or A nucletide expansion in four dominant groups of trinucleotides which in coding parts of genomes represent start and stop codons. Such extended clusters are referred to as start/stop codon-like trinucleotides (CLTs). It is shown that what significantly distinguishes alpha satellite sequences in human centromere and pericentromere regions from non-alpha satellite HORs and from no-repeat noncoding sequences are the specificity and the level of extensions of start/stop CLTs, with dominant contribution from the stop-TGA CLTs. Successive multiplications of T nucleotides in stop-TGA CLTs, and to somewhat smaller extent due to multiplication of $\mathrm{A} \mathrm{nu}-$ cleotides, leads to the poly-T class of all human alpha satellite HORs while the monomeric alpha satellites can be of poly-T or of poly-A class. The basic role of stop-TGA CLT is that the poly-T stretch preceding $G$ nucleotide and the poly-A stretch following it (poly-T-G-poly-A) exhaust about $30 \%$ of all $\mathrm{T}$ and $\mathrm{A}$ nucleotides in alpha satellites. Distributions of extended and non-extended CLTs within alpha satellites do not appear randomly, but form a well organized structure. In this way, this work opens a question of information potential of the new "CLT-language".

The CLT framework can provide also a structural function as nucleosome positioning patterns, giving a possible explanation of alpha satellite segmentation into two wrapping sequences around the nucleosome and a linker.

It is a question now what could be in centromeres a biological function of so sophisticated CLT structure, resembling to a new language of DNA with an additional information potential, because if there is a goal only to achieve the strength of centromere, this purpose could be satisfied in simpler ways. It could be hypothesized that CLTs in such a well organized form as in alpha satellites may be the related to regulative role in the genome, with noncoding RNAs transmitting the information signal to the gene network. This intriguing point might be of interest for future experimental investigations. 
Supplementary Materials. - Supporting informations to the paper (Figures S1 and S2; Tables S1 to S3) are enclosed to the electronic version of the article. These data can be found on the website of Croatica Chemica Acta (http://public.carnet.hr/ccacaa).

Acknowledgements. The authors thank Chris Tyler-Smith for suggestion to look for possible new classifications of alpha satellites, and to anonymous reviewer for very useful comments.

\section{REFERENCES}

1. F. Jacob and J. Monod, J. Mol. Biol. 3 (1961) 318-356.

2. R. J. Britten and E. H. Davidson, Science 165 (1969) 349-357.

3. M. C. King and A. C. Wilson, Science 188 (1975) 107-116.

4. L. A. Pennacchio, N. Ahituv, A. M. Moses, S. Prabhakar, M. A. Nobrega, M. Shoukry, S. Minovitsky, I. Dubchak, A. Holt, K. D. Lewis, I. Plajzer-Fick, J. Akiyama, S. De Val, V. Afzal, B. L. Black, O. Couronne, M. B. Eisen, A. Visel, and E. M. Rubin, Nature 444 (2006) 499-502.

5. G. A. Wray and C. C. Babbitt, Science 321 (2008) 1300-1301.

6. D. A. Garfield and G. A. Wray, BioScience 60 (2010) 15-23.

7. J. P. Noonan and A. S. McCallion, Annu. Rev. Genomics Hum. Genet. 11 (2010) 1-23.

8. E. N. Trifonov, Comput. Chem. 17 (1993) 27-31.

9. L. Manuelidis and J. C. Wu, Nature 276 (1978) 92-94.

10. H. F. Willard, Amer. J. Hum. Genet. 37 (1985) 524-532.

11. C. Tyler-Smith, Development 101 (1985) 93-100.

12. C. Tyler-Smith and W. R. A. Brown, J. Mol. Biol. 195 (1987) 457-470.

13. P. E. Warburton and H. F. Willard, Evolution of centromeric alpha satellite DNA: molecular organization within and between human and primate chromosomes, in: M. Jackson, T. Strachan, and G. Dover (Eds.), Human Genome Evolution, BIOS Scientific, Oxford, 1996.

14. M. K. Rudd, G. A. Wray, and H. F. Willard, Genome Res. 16 (2006) 88-96.

15. R. Nussinov, Nucleic Acids Res. 12 (1984) 1749-1763.
16. E. Beutler, T. Gelbart, J. Han, J. A. Koziol, and B. Beutler, Proc. Natl. Acad. Sci. USA 86 (1989) 192-196.

17. S. Karlin and J. Mrazek, Proc. Natl. Acad. Sci. USA 94 (1997) 10227-10232.

18. S. Karlin, Curr. Opin. Microbiol. 1 (1998) 598-610.

19. P. F. Arndt, C. B. Burge, and T. Hwa, J. Comput. Biol. 10 (2003) 313-322.

20. C. K. Peng, S. V. Buldyrev, A. L. Goldberger, S. Havlin, F. Sciortino, M. Simons, and H. E. Stanley, Nature 356 (1992) 168-170.

21. Y. Almirantis and A. Provata, Bull. Math. Biol. 59 (1997) $975-$ 992.

22. E. Segal and J. Widom, Curr. Opin. Struct. Biol. 19 (2009) 6571.

23. C. K. Collings, A. G. Fernandez, C. G. Pitschka, T. B. Hawkins, and J. N. Anderson, PLoS One 5(6) (2010) e10933.

24. G. Levinson, and G. A. Gutman, Mol. Biol. Evol. 4 (1987) 203 221.

25. M. Rosandić, V. Paar, and I. Basar, J. Theor. Biol. 221 (2003) 29-37.

26. M. Rosandić, V. Paar, M. Glunčić, I. Basar, N. Pavin, Croat. Med. J. 44 (2003) 386-403.

27. V. Paar, N. Pavin, I. Basar, M. Rosandić, I. Luketin, and S. Durajlija Žinić, Croat. Chem. Acta 77 (2004) 73-81.

28. V. Paar, N. Pavin, M. Rosandić, M. Glunčić, I. Basar, R. Pezer, and S. Durajlija Žinić, Bioinformatics 21 (2005) 846-852.

29. M. Rosandić, V. Paar, I. Basar, M. Glunčić, N. Pavin, and I. Pilaš, Chromosome Res. 14 (2006) 735-753.

30. V. Paar, I. Basar, M. Rosandić, and M. Glunčić, Curr. Genomics 8 (2007) 93-111.

31. M. Rosandić, M. Glunčić, V. Paar, and I. Basar, J. Theor. Biol. 254 (2008) 555-560.

32. V. Paar, M. Glunčić, I. Basar, M. Rosandić, P. Paar, and M. Cvitković, J. Mol. Evol. 72 (2011) 34-55.

33. V. Paar, M. Glunčić, M. Rosandić, I. Basar, and I. Vlahović, Mol. Biol. Evol. 28 (2011) 1877-1892.

34. A. E. Rapaport, Z. M. Frenkel, and E. N. Trifonov, J Biomol Structure \& Dynamics 4 (2011) 567-574.

35. Z. M. Frenkel, T. Bettecken, and E. N. Trifonov, BMC Genomics 12 (2011) 203. 